\title{
Practical considerations for territorial perfusion mapping in the cerebral circulation using super-selective pseudo-continuous arterial spin labeling
}

\author{
Jonas Schollenberger ${ }^{1}$ (iD \\ C. Alberto Figueroa ${ }^{1,2}$ (iD \\ Luis Hernandez-Garcia ${ }^{1,3}$ \\ ${ }^{1}$ Department of Biomedical Engineering, University of Michigan, Ann Arbor, Michigan \\ ${ }^{2}$ Department of Surgery, University of Michigan, Ann Arbor, Michigan \\ ${ }^{3}$ FMRI Laboratory, University of Michigan, Ann Arbor, Michigan
}

Jon-Fredrik Nielsen $^{1,3}$

\section{Correspondence}

Jonas Schollenberger, Department of Biomedical Engineering, University of Michigan, North Campus Research Complex, 2800 Plymouth Road, Ann Arbor, MI 48109.

Email: scjonas@umich.edu

\section{Funding information}

The German Academic Exchange Service

(DAAD); National Institutes of Health

(R21EB021562); and the Edward B.

Diethrich Professorship.
Purpose: This paper discusses several challenges faced by super-selective pseudo-continuous arterial spin labeling, which is used to quantify territorial perfusion in the cerebral circulation. The effects of off-resonance, pulsatility, vessel movement, and label rotation scheme are investigated, and methods to maximize labeling efficiency and overall image quality are evaluated. A strategy to calculate the territorial perfusion fractions of individual vessels is proposed.

Methods: The effects of off-resonance, label rotation scheme, and vessel movement on labeling efficiency were simulated. Two off-resonance compensation strategies (multiphase prescan, field map), cardiac triggering, and vessel movement were studied in vivo in a group of 10 subjects. Subsequently, a territorial perfusion fraction map was acquired in 2 subjects based on the mean vessel labeling efficiency.

Results: Multiphase calibration provided the highest labeling efficiency $(P=.002)$ followed by the field map compensation $(P=.037)$ compared with the uncompensated acquisition. Cardiac triggering resulted in a qualitative improvement of the image and an increase in signal contrast between the perfusion territory and the surrounding tissue $(P=.010)$ but failed to show a significant change in temporal and spatial SNR. The constant clockwise label rotation scheme yielded the highest labeling efficiency. Significant vessel movement ( $>2 \mathrm{~mm}$ according to simulations) was observed in $50 \%$ of subjects. The measured territorial perfusion fractions showed good agreement with anatomical data.

Conclusion: Optimized labeling efficiency resulted in increased image quality and accuracy of territorial perfusion fraction maps. Labeling efficiency depends critically on off-resonance calibration, cardiac triggering, optimal label rotation scheme, and vessel location tracking.

\section{K E Y W O R D S}

arterial spin labeling, cardiac triggering, labeling efficiency, off-resonance, super-selective, territorial perfusion 


\section{1 | INTRODUCTION}

Perfusion measurements of vascular territories in the brain offer critical clinical information on cerebrovascular function. In the presence of steno-occlusive cerebrovascular disease, vascular territory maps can be used to identify and evaluate collateral flow pathways. ${ }^{1,2}$ In cryptogenic stroke, blood supply knowledge of individual arteries to different parts of the brain can help identify the origin of the stroke and guide treatment. ${ }^{3}$ The current clinical gold standard for measuring brain vascular territories is digital subtraction angiography, an invasive, nonquantitative procedure that relies on the injection of a contrast agent, which increases the risk of neurologic complications. ${ }^{4}$

In the last decade, the use of MRI arterial spin labeling (ASL) has gained popularity as a noninvasive and quantitative alternative for measuring brain tissue perfusion. Pseudo-continuous arterial spin labeling (pCASL) is the current preferred inversion scheme, ${ }^{5}$ in which blood spins flowing through a label plane are continuously inverted to generate a bolus of label that can be measured downstream in the tissue after a short transit time. More recently, the pCASL scheme was extended to include vessel-selective labeling to map perfusion territories. Vessel-selective labeling generally uses additional in-plane gradient blips (perpendicular to the slice-selective gradient) in between RF pulses and the tracking of the accumulated phase at the target location for the next RF pulse. In vessel-encoded pCASL, ${ }^{6}$ combinations of vessel pairs are placed in label and control condition by in-plane gradients with a constant amplitude to generate a $\pi$-phase shift between vessel pairs. Contributions from individual vessels are estimated by acquiring multiple combinations based on a Hadamard encoding strategy. To facilitate labeling of individual vessels and to avoid lengthy postprocessing procedures, Helle et $\mathrm{al}^{7}$ and Dai et $\mathrm{al}^{8}$ developed super-selective pCASL (SS-pCASL). By applying gradient blips with time-varying amplitudes, the SS-pCASL scheme generates a circular label spot with adaptable center location and size. Although the ability to label intracranial arteries is one of the major advantages of SS-pCASL, the focus of this work is on labeling in the neck vessels. There is considerable clinical interest in mapping the perfusion territories of the main brain-feeding arteries due to the high anatomical variability of the circle of Willis. ${ }^{9}{ }^{90}$ However, many of the issues and solutions investigated are also applicable to intracranial territorial mapping as well as to vessel-encoded pCASL.

While the feasibility of SS-pCASL has been shown previously in a number of clinical applications, ${ }^{1,11-13}$ SNR still remains a challenge. ${ }^{14}$ Pseudo-continuous labeling is well known to be a low SNR technique. In SS-pCASL, SNR is further reduced because of inherently lower labeling efficiency, ${ }^{7}$ particularly noticeable in low-flow vessels such as the vertebral arteries. A reduction in labeling efficiency can result in the erroneous conclusion that an artery is not contributing to the perfusion of a region in the brain. Labeling efficiency can vary significantly within a scan session, between vessels, and across subjects. Consequently, there is a need to maximize labeling efficiency and to assess efficiency differences between vessels, to produce accurate, quantitative territorial perfusion maps. These challenges, together with the overall availability of the sequence, must be addressed for SS-pCASL to become mainstream. In the following, the primary causes of reduction in labeling efficiency and SNR, as well as our strategies to mitigate them, are outlined.

\section{1 | Field inhomogeneity (off-resonance)}

In the presence of off-resonance, the mismatch between the applied RF phase increment and the phase gain experienced by the spins results in a weakened spin lock, thereby reducing the efficiency of the flow-driven adiabatic inversion. Although rarely used in current publications, a number of off-resonance compensation strategies for nonselective pCASL have been proposed. ${ }^{15-17}$ Here, we evaluate 2 compensation strategies for SS-pCASL, a multiphase prescan and a field map acquisition, to estimate the optimal off-resonance phase compensation. While the field map measures field inhomogeneity directly, the multiphase prescan estimates the off-resonance indirectly from a set of perfusion images acquired over a range of RF-phase correction increments.

\section{2 | Pulsatility}

In pCASL, label and control images are acquired sequentially in a fixed interval (TR) and therefore at different time points in the cardiac cycle. Variations in blood flow during the label period can affect labeling efficiency. Furthermore, differences in the blood volume in the cerebral arteries at the time of the acquisition can lead to significant artifacts when subtracting the label from the control images. Here, we explore the use of cardiac-triggered SS-pCASL to reduce the effects of pulsatility based on the scheme for standard pCASL by Li et al. ${ }^{18}$

\section{3 | Label rotation scheme}

The circular label spot in SS-pCASL is created by applying time-varying in-plane gradient blips, which create a set of rotating inversion bands. The rotation scheme is determined by the time course of the in-plane gradients. Previously proposed label rotation schemes have shown a significant influence on labeling efficiency. ${ }^{7}$ Based on Bloch simulations, we compare the labeling efficiency of multiple label rotation schemes in the absence and presence of off-resonance. 


\subsection{Label spot size and position}

The success of SS-pCASL depends on accurate positioning of the label spot on the vessel of interest. Any mismatch can result in a significant reduction in labeling efficiency. The effects of vessel movement are explored using Bloch simulations and compared with in vivo vessel movement measurements.

This work is intended as a practical guideline for acquiring high-quality territorial perfusion fraction maps with SSpCASL. Toward that goal, we first investigate the effects of off-resonance, pulsatility, vessel movement, and rotation scheme on SS-pCASL labeling efficiency and overall image quality. We evaluate strategies to mitigate different sources of error based on practicality and effectiveness. Finally, we present a strategy to estimate the territorial perfusion fraction for individual neck arteries based on direct measurements of labeling efficiency.

\section{2 | METHODS}

\section{1 | Bloch simulations}

Simulations were performed in MATLAB (MathWorks, Natick, MA) to investigate the effects of off-resonance, label rotation scheme, and vessel location on labeling efficiency. We simulated the inversion of the longitudinal magnetization of spins flowing through the label plane subject to SS-pCASL RF and gradient pulses. The flow of spins was modeled in a 4-cm slab centered around the label plane. Based on the spatial mean velocity in the internal carotid artery (ICA) and vertebral artery (see phase-contrast MRI data from study 2 in section 2.2), a parabolic velocity profile was generated for each of the 20 acquired temporal phases. The parabolic velocity profiles were discretized with a grid containing 30 points in the radial direction. For each point of the profile, Bloch simulations were repeated for all cardiac phases. Based on the balanced version of PCASL, "label" and "control" pulse trains were simulated with the same gradient waveform but with a $180^{\circ} \mathrm{RF}$ phase shift between RF pulses in the control condition. Labeling efficiency of each spin was calculated as

$$
\alpha=\left(M_{Z, \text { Control }}-M_{Z, \text { Label }}\right) /\left(2 M_{Z, \text { Control }}\right),
$$

where $M_{Z, \text { Control }}$ and $M_{Z, \text { Label }}$ are the longitudinal spin magnetizations at the end of the simulated slab for the control and label conditions, respectively. The labeling efficiency was then averaged over the cardiac cycle and subsequently velocity-weighted over the vessel cross section to yield a mean labeling efficiency. The parameters of the label train were set to match in vivo parameters (see section 2.2). The amplitudes of the in-plane gradients are determined by a design-specific rotation scheme. We focused on 3 schemes: constant clockwise, fully random, and pseudo-random rotation. ${ }^{7}$ All schemes were based on 32 evenly distributed angular gradient positions and a constant effective in-plane gradient $G_{x y, k}=\sqrt[2]{G_{x, k}^{2}+G_{y, k}^{2}}=4 \mathrm{mT} / \mathrm{m}$, which corresponds to a label spot size of $18 \mathrm{~mm}(\mathrm{FWHM})$. The values of $\mathrm{T}_{1}$ and $\mathrm{T}_{2}$ were set to $1650 \mathrm{~ms}$ and $250 \mathrm{~ms}$, respectively.

Mean labeling efficiency was evaluated for different amounts of off-resonance, ranging from $0 \mathrm{~Hz}$ to $400 \mathrm{~Hz}$. The 3 label rotation schemes were simulated and compared with a nonselective label scheme. The effects of vessel movement were simulated by moving the center of the vessel cross section out of the label spot. Mean labeling efficiency was evaluated over a range of $0-10 \mathrm{~mm}$ vessel movement.

\section{2 | In vivo imaging}

Two in vivo studies were performed on a total of 12 healthy subjects ( 9 males, 3 females, mean age of 27.8). The study protocol was approved by the local internal review board, and written consent was obtained from each participant. All scans were performed at $3 \mathrm{~T}$ (MR750; GE Healthcare, Waukesha, WI) with a 32-channel receive-only head coil.

\subsection{1 | Study 1: Optimization of labeling efficiency}

This study included 10 volunteers. The protocol started with a 2D time of flight (TOF) of the neck to determine the optimal position of the label plane and the location of the arteries. A position slightly above the carotid bifurcation was selected, where both carotid and vertebral arteries run perpendicular to the label plane and the distance between vessels is maximal. The sequence parameters are TR/TE $=12.7 / 2.4 \mathrm{~ms}$, voxel size $=0.43 \times 0.43 \times 4 \mathrm{~mm}^{3}, 20$ slices, and acquisition time $=$ 47 seconds. The image volume, not including the region of the label plane, was shimmed only once before the first pCASL sequence using the standard GE Healthcare prescan procedure. The table position was kept fixed throughout the perfusion scans to avoid any change in the magnetic field.

\section{Off-resonance calibration}

Off-resonance was investigated by acquiring 3 sets of SSpCASL images of the left ICA, each with a different offresonance calibration. The calibration strategies consisted of a multiphase prescan, a field map, and a scan without calibration. We performed the 3 calibration strategies in randomized order, each followed by a cardiac-triggered SS-pCASL scan (details to follow). In the multiphase calibration, a nonselective pCASL perfusion prescan $\left(G_{x y}=0 \mathrm{mT} / \mathrm{m}\right)$ was acquired with multiple phase corrections added to the RF pulse train. 
The RF phase correction was increased in steps of $0.4 \mathrm{rad}$, covering a range of $[-3.2,3.2 \mathrm{rad}]$ between acquisitions of each label/control pair. The sequence consisted of a singleshot spiral acquisition (slices $=16$, voxel size $=3.75 \times 3.75 \times$ $7 \mathrm{~mm}^{3}, \mathrm{TR} / \mathrm{TE}=4000 / 4 \mathrm{~ms}$, label duration $=1800 \mathrm{~ms}$, postlabeling delay $=1400 \mathrm{~ms}$, number of frames $=34(17$ phase increments), background suppression, acquisition time = 2 minutes 40 seconds). The slice prescription and the remaining sequence parameters were identical to the SS-pCASL scan (details to follow). The optimal off-resonance phase correction was estimated on the fly by subtracting pairs of control and label, masking a slice in the center of the image volume, averaging the signal intensity for each phase, and finding the maximum of a least-squares curve fit with a sine function. Next, a field map was collected in the same location as the pCASL label plane to measure the off-resonance directly. The acquisition consisted of a spoiled gradient-echo single slice $\left(\mathrm{TR}=18 \mathrm{~ms}\right.$, voxel size $=0.85 \times 0.85 \times 4 \mathrm{~mm}^{3}$, flip angle $=20^{\circ}$, and a $\Delta \mathrm{TE}=2.5 \mathrm{~ms}$, which corresponds to an off-resonance frequency range of $\pm 200 \mathrm{~Hz}$ ). The phase correction was estimated by manually drawing a region of interest (ROI) around the vessel of interest and calculating the average off-resonance. The vessel itself was excluded from the ROI to avoid flow effects.

\section{Cardiac triggering}

Two sets of SS-pCASL images were collected with and without cardiac triggering, using the phase calibration of the multiphase prescan. For this comparison, the dominant vertebral artery was labeled, and the pulsatility artifacts, which are most dominant in the middle cerebral artery, were observed outside the posterior perfusion territory. In the cardiac-triggered SSpCASL perfusion scan, the balanced label/control pulse train was applied for $1800 \mathrm{~ms}$ (in-plane gradient amplitude $G_{x y}=$ $4 \mathrm{mT} / \mathrm{m}$, slice-selective gradient amplitude $G_{z}=6 \mathrm{mT} / \mathrm{m}$, average slice-selective gradient $G_{z, \text { ave }}=0.6 \mathrm{mT} / \mathrm{m}, 1$-ms time gap between RF pulses). After a postlabeling delay of $1800 \mathrm{~ms}, 16$ slices were collected with a 2 -shot spiral acquisition scheme $\left(\right.$ voxel size $\left.=3.75 \times 3.75 \times 7 \mathrm{~mm}^{3}\right)$. Six subtraction image pairs were collected. The background suppression scheme included a saturation pulse right before the start of the RF label train and 2 adiabatic inversion pulses during the transit delay, followed by a fat suppression pulse. A cardiac-triggered scheme based on Li et al ${ }^{18}$ was used to trigger the start of the saturation pulse, as measured by a peripheral pulse oximeter, attached to the subject's index finger. After a minimum TR of $4400 \mathrm{~ms}$, the sequence waited for a trigger to start the next label period. A proton density image was collected at the beginning of each perfusion scan. The total acquisition time for the perfusion scan was 2 minutes 45 seconds \pm 10 seconds. In the nontriggered scan, the TR was fixed at $4400 \mathrm{~ms}$. All other sequence parameters were kept identical to the cardiactriggered scan. A nonselective perfusion scan with the cardiactriggered, balanced pCASL scheme was collected as well.
Vessel movement

A single-slice 2D TOF was collected with the same TOF parameters in the label plane before each perfusion scan to detect any subject movement and to update the vessel location needed for accurate placement of the label spot (acquisition time $=2$ seconds).

\subsection{2 | Study 2: Territorial perfusion fraction map}

Territorial perfusion fraction maps were obtained in 2 additional healthy volunteers based on the results of study 1 . The protocol started with a 2D TOF and a multiphase prescan followed by a cardiac-triggered SS-pCASL of both carotid and vertebral arteries. The cardiac-triggered SS-pCASL was then adjusted to estimate labeling efficiency of each vessel directly above the label plane. Two image slices with a $18-\mathrm{cm}$ FOV and 3-mm slice thickness were positioned approximately $2 \mathrm{~cm}$ above the label plane. The label time was $500 \mathrm{~ms}$ and the postlabeling delay was $10 \mathrm{~ms}$. Background suppression was turned off except for the fat-suppression pulse. For each vessel, 2 pairs of label and control images were acquired with 4 interleaved shots using the same off-resonance compensation. All 4 arteries were collected sequentially in a single scan, resulting in a total acquisition time of about 2 minutes.

At the end of the protocol, phase-contrast MRI was acquired with the same slice prescription as the 2 slices used for labeling efficiency (gradient echo, TR/TE $=5.3 / 3.1 \mathrm{~ms}$, voxel size $=0.43 \times 0.43 \times 4 \mathrm{~mm}^{3}$, velocity encoding $=$ $100 \mathrm{~cm} / \mathrm{s}$, phase-encoding perpendicular to slice, 20 cardiac phases). Additionally, a 3D TOF of the circle of Willis was obtained for anatomical reference (gradient spoiled echo, TR/ $\mathrm{TE}=21 / 2.5 \mathrm{~ms}$, voxel size $=0.43 \times 0.43 \times 1.5 \mathrm{~mm}^{3}, 2$ slabs with a total of 118 slices, autocalibration reconstruction for Cartesian sampling acceleration factor $=2$, acquisition time $=$ 2 minutes 48 seconds).

\subsection{Image postprocessing and analysis}

Image postprocessing and analysis were performed in MATLAB. All SS-pCASL images were reconstructed to a $128 \times 128$ resolution using zero-padding in k-space. Label and control images were averaged and subtracted.

\subsection{1 | Study 1: Optimization of labeling efficiency}

\section{Off-resonance calibration}

Perfusion images were first normalized by the proton density image. A relative labeling efficiency was calculated by dividing the vessel-selective by the nonselective perfusion images and masking the territory of the left ICA. The mask, which included gray and white matter, was created 
by manually defining the outline of the left ICA perfusion territory based on the mean vessel-selective perfusion image. Finally, a histogram of the relative labeling efficiency was least-squares fitted with a Gaussian function to estimate the mean relative labeling efficiency. The difference between calibration strategies was analyzed using a nonparametric Wilcoxon signed rank test with a significance threshold of 0.05 .

\section{Cardiac triggering}

A quantitative comparison of the cardiac-triggered and nontriggered images was performed by selecting a single slice in the center of the volume and creating 2 ROIs: an "inside" ROI of the vertebral perfusion territory and an "outside" ROI of the small vessels distal to the middle cerebral artery, to capture arterial pulsatility artifacts. We calculated a spatial SNR and a temporal SNR in both ROIs. Spatial SNR was defined as the mean signal over the spatial SD outside the brain. Temporal SNR was calculated as the mean signal over the temporal SD within the ROI based on 6 pairwise subtraction images. Successful reduction of pulsatility artifacts was characterized by high SNR in the inside ROI and low SNR in the outside ROI. Furthermore, a contrast-tonoise ratio was defined as the difference between the 2 ROIs divided by the temporal SD outside the brain. A statistical comparison was performed with a nonparametric Wilcoxon signed rank test with a significance threshold of 0.05 . Additionally, a qualitative comparison was conducted by 4 expert observers. Each observer rated the pairs of cardiactriggered and nontriggered images without prior knowledge of the acquisition method. Image quality was assessed by the delineation of the perfusion territory and by the amount of pulsatility artifacts outside the perfusion territory.

\section{Vessel movement}

Vessel movement was quantified by measuring the vessel center locations in each acquired 2D TOF image and calculating the absolute distance to the initial location at the beginning of the protocol.

\subsection{2 | Study 2: Territorial perfusion fraction map}

\section{Labeling efficiency of individual arteries}

The vessel-averaged labeling efficiency was obtained by first calculating the labeling efficiency map $\alpha_{\text {meas }}$ based on the complex reconstructed SS-pCASL images above the label plane (Equation 1). The true labeling efficiency was calculated by compensating for signal loss due to $T_{1}$ decay during transit on a voxel-by-voxel basis according to

$$
\alpha_{\text {true }}=\alpha_{\text {meas }} \cdot \exp \left(\Delta_{s l} v^{-1} T_{1, \text { art }}^{-1}\right)
$$

where $\Delta_{s l}$ is the distance between label plane and the image slice, chosen such that all 4 vessels are intersected as perpendicularly as possible. The cardiac-averaged velocity field $v$ was acquired with phase-contrast MRI and coregistered to the mean subtraction image above the label plane. The $\mathrm{T}_{1}$ relaxation in arterial blood was set to $\mathrm{T}_{1, \text { art }}=1650 \mathrm{~ms}$. Finally, each vessel was masked, and a velocity-weighted average labeling efficiency was calculated.

For validation, a relative labeling efficiency was defined as the ratio of vessel-selective to nonselective vessel-averaged labeling efficiency. The relative labeling efficiency based on the measurements in the neck was then compared with the relative labeling efficiency based on the measured perfusion signal in the carotid territories of the brain (see section 2.3).

\section{Territorial Perfusion fraction map}

Vessel-selective perfusion images were first scaled according to their average labeling efficiency. A median filter with a $4 \times 4$ window was applied to remove noise. The perfusion fraction map was calculated by dividing the efficiency-scaled vessel-selective images by the total perfusion on a voxel-byvoxel basis. Total perfusion was defined as the sum of all efficiency-scaled vessel-selective perfusion images, to yield a fraction sum of 1 . The resulting combined perfusion image was then visually compared with the measured perfusion image obtained with the nonselective method to ensure that all main feeding arteries were included. For reference, a standard color-coded territorial perfusion map was created by assigning each voxel a color based on the highest contributing artery.

\section{3 | RESULTS}

\section{1 | Off-resonance calibration}

Figure 1 shows the simulated mean vessel labeling efficiency for different label rotation schemes over a range of off-resonance frequencies. The labeling efficiency is robust for small amounts of off-resonance, but it significantly decreases for larger amounts of off-resonance. For an off-resonance frequency greater than $250 \mathrm{~Hz}$, which corresponds to a phase accumulation of $\pi / 2$, the order of label and control switch (negative mean labeling efficiency). Overall, the vessel-selective label schemes were more prone to a reduction in labeling efficiency due to offresonance compared with the nonselective label scheme. The constant clockwise rotation scheme showed the highest labeling efficiency for small amounts of off-resonance. However, for larger amounts, the labeling efficiency of the constant clockwise scheme decreased faster compared with other label rotation schemes. The pseudo-random scheme offered the best results when large amounts of offresonance are present but showed diminished maximal labeling efficiency at small amounts of off-resonance. The 


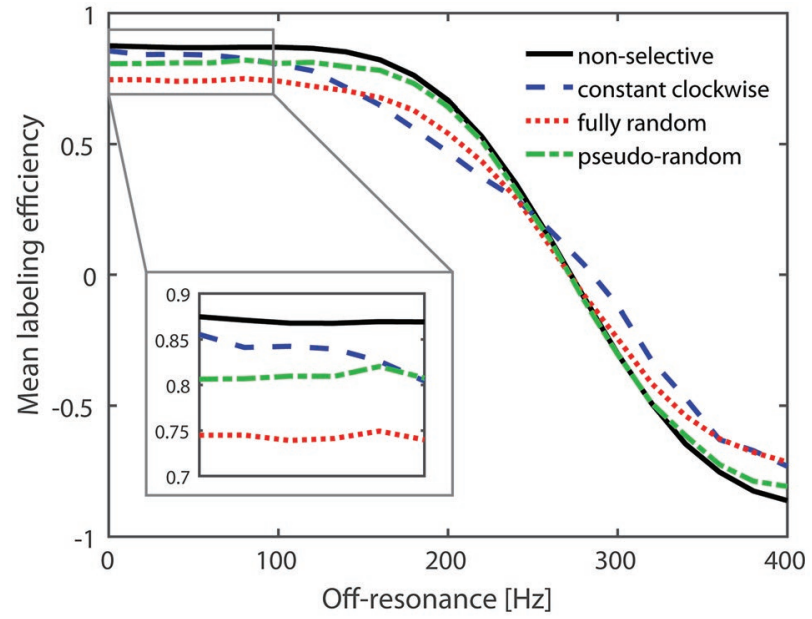

F I G URE 1 Simulated mean labeling efficiency in the presence of off-resonance for different label rotation schemes. The close-up shows the difference in labeling efficiency between label rotation schemes for small amounts of off-resonance

fully random scheme showed overall the lowest labeling efficiency. The constant clockwise scheme was chosen for all ensuing in vivo experiments, given its superior labeling efficiency in combination with the proposed off-resonance compensation method.

In the first part of study 1 , an in vivo comparison of 2 offresonance calibration strategies was performed. Figure 2 illustrates the calculation of the off-resonance phase compensation based on a multiphase prescan (Figure 2A) and a field map (Figure 2B). The results of the off-resonance calibration comparison are summarized in Figure 3. Both the multiphase and field map-calibrated SS-pCASL scans yielded statistically significant increases in labeling efficiency (Wilcoxon signed rank test, $P=.002$ and $P=.037$, respectively) compared with the noncalibrated scan (Figure $3 \mathrm{~A})$. The multiphase calibration consistently outperformed the field map calibration (Wilcoxon signed rank test, $P=$ .002). The left ICA perfusion territory of 2 subjects is illustrated in Figure 3B for each calibration strategy. For subject 1 , both field map-calibrated and noncalibrated scans had significantly reduced labeling efficiency, which resulted in difficulties to distinguish between perfusion signal and background noise. The multiphase-calibrated scan provided a clear image of the perfusion territory. Subject 2 presented with an anatomical variation of the circle of Willis, where the territory of the right anterior cerebral artery was perfused by the left ICA. This is an important perfusion detail that was difficult to identify in the noncalibrated scan. Both the multiphase and field map-calibrated scan achieved sufficient quality to identify the perfusion territory as well as capture the anatomical variation. The measured off-resonance phase corrections based on the multiphase prescan and the field map are plotted in Figure 4. A linear regression model revealed a constant offset between the off-resonance phase obtained from the field map and the multiphase prescan.

\section{(A) multi-phase pre-scan}
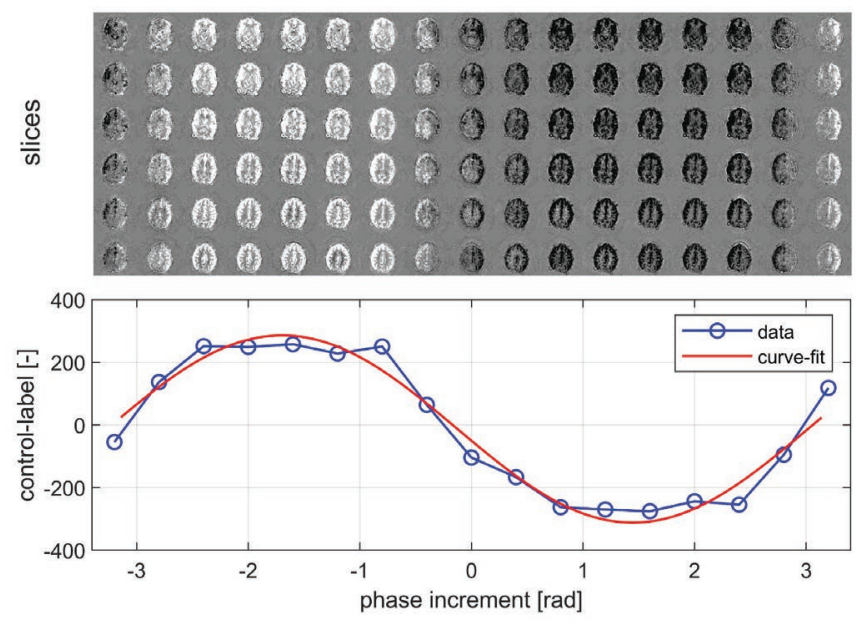

(B) field map

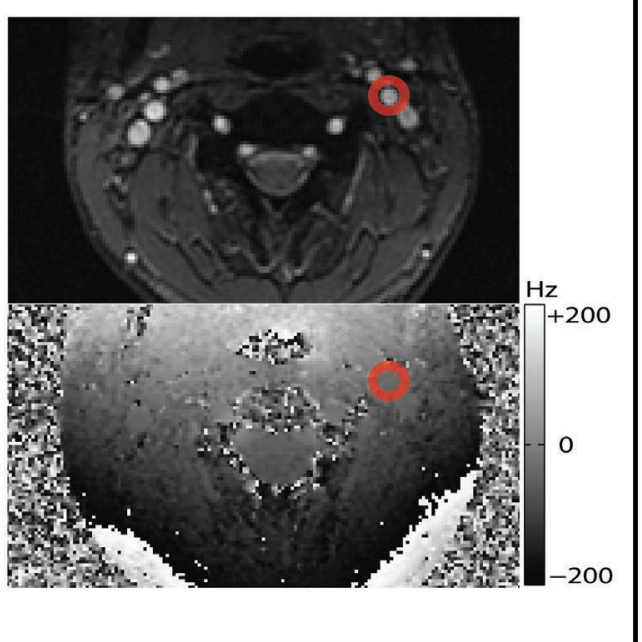

F I G U R E 2 Off-resonance calibration. A, In the multiphase prescan, the perfusion signal is measured for different amounts of added phase. Each column in the top image represents 1 phase increment; the rows correspond to different slice positions. The optimal phase correction was determined by a least-squares curve fit with a sine function. The blue line represents the average signal for each phase increment, and the red line the curve fit. B, Based on the acquisition of a field map in the same location as the pseudo-continuous arterial spin labeling (pCASL) label plane, off-resonance was measured around the vessel of interest. First, a region of interest (ROI) was drawn manually in the magnitude image (top) and then applied to the frequency image (bottom) 


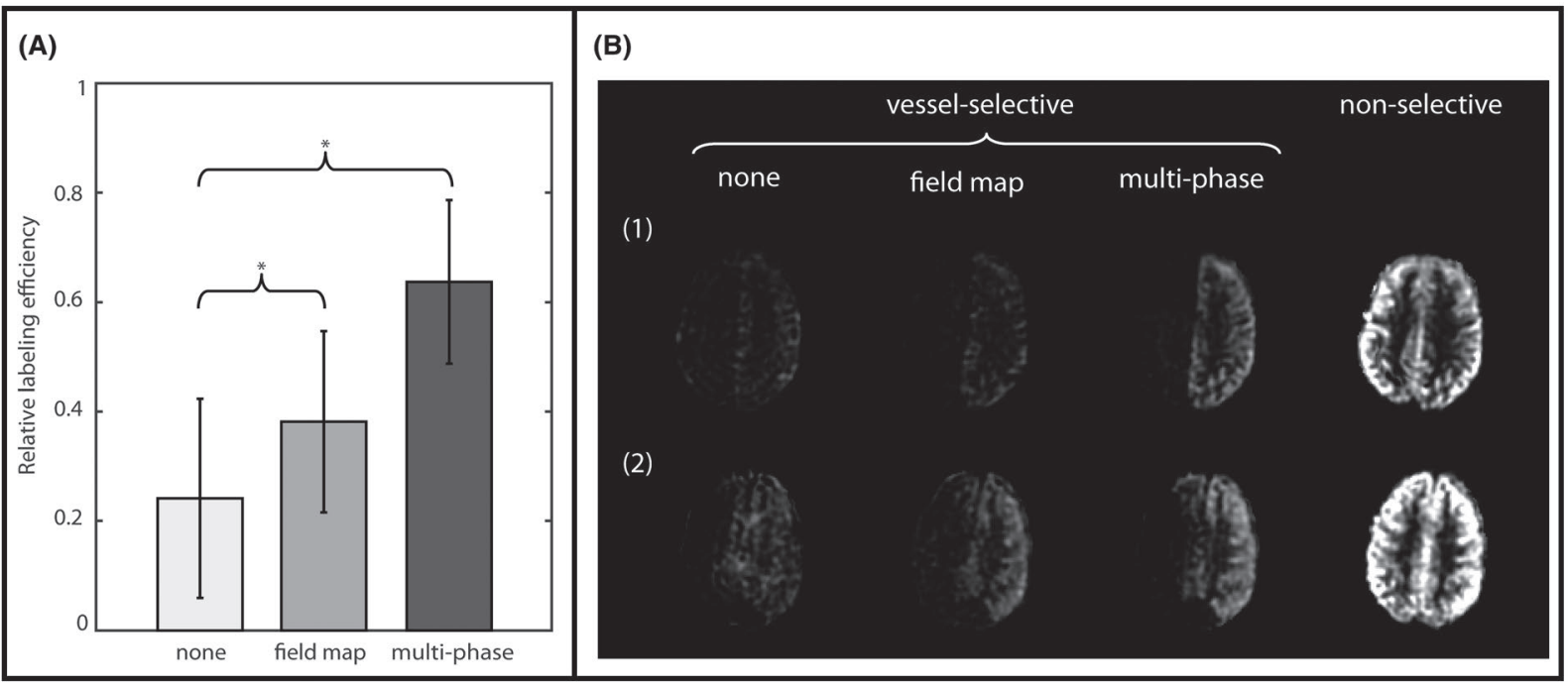

F I G U RE 3 A, Quantitative comparison of the average relative labeling efficiency for 3 off-resonance calibration approaches (i.e., multiphase prescan, field map, no calibration) over all subjects. The asterisk (*) represents a value of $P<.05$ with the Wilcoxon signed rank test. The multiphase calibrated scan consistently produced the highest signal, followed by the field map-calibrated and the noncalibrated scan.

B, Left internal carotid artery (ICA) perfusion territory of 2 subjects for different off-resonance calibration strategies compared with a nonselective perfusion scan. Overall, the multiphase calibrated scan yielded the highest amount of signal. Subject 1 yielded overall low perfusion signal for the field map and the noncalibrated scans; only multiphase calibration resulted in an adequate representation of the territory. The same pattern was observed in subject 2 , who presented with anterior collateral flow, best visible in the multiphase calibrated scan

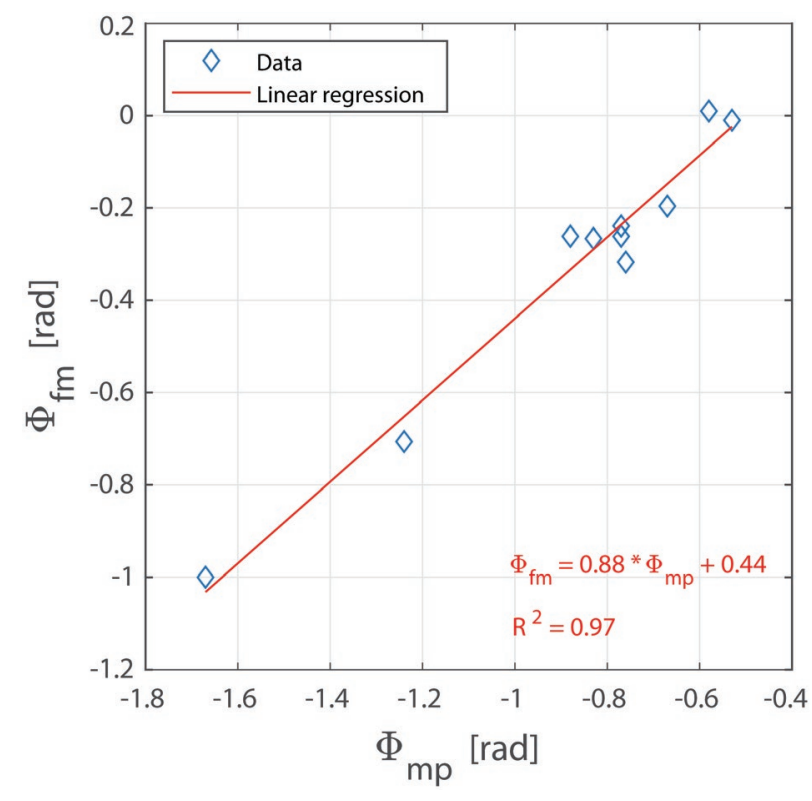

F I G URE 4 Pairwise comparison of the off-resonance phase measured with the multiphase prescan (mp) and the field map (fm). Data were fitted with a linear regression model

\section{2 | Cardiac triggering}

The efficacy of cardiac triggering on reducing pulsatility effects and improving overall image quality was investigated in study 1. A qualitative comparison of the cardiac-triggered and nontriggered mean subtraction images resulted, on average, in improved image quality with cardiac triggering

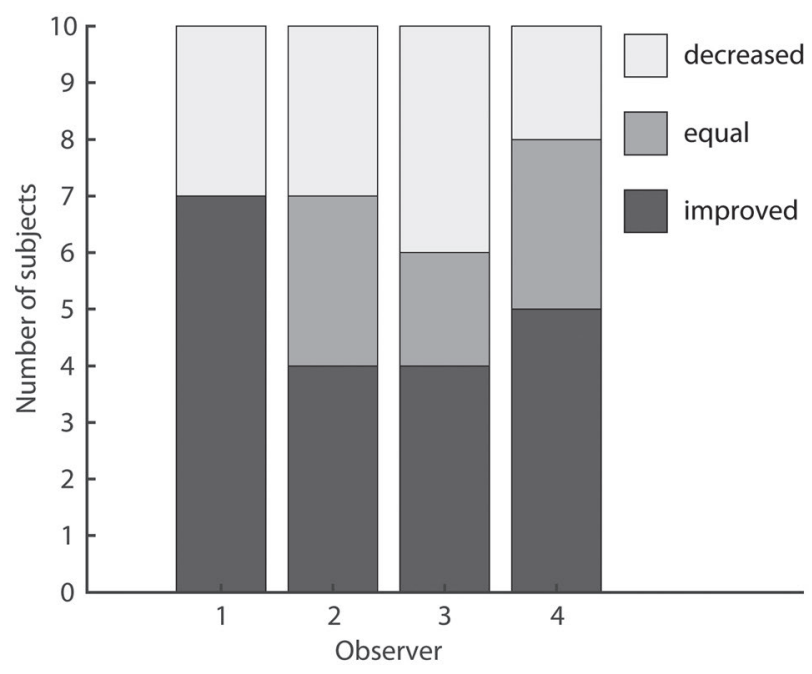

F IG URE 5 Qualitative comparison of cardiac-triggered and standard nontriggered super-selective pCASL. Changes in the image quality with cardiac triggering were assessed by 4 expert observers and rated as improved, equal, or decreased

(Figure 5). A subject with improved outcome is shown in Figure 6. The pairwise subtractions of the nontriggered acquisition displayed pulsatility artifacts around the middle and anterior cerebral arteries (white arrows), whereas the cardiac-triggered acquisition showed a significant reduction in pulsatility artifacts. A quantitative analysis of the cardiac triggering performance is shown in Figure 7. Both spatial SNR and temporal SNR did not result in a significant change 
FIG URE 6 Pairwise and mean subtraction images for a nontriggered and cardiac-triggered perfusion scan of the dominant vertebral artery (VA) in the same subject. White arrows indicate pulsatility artifacts in the middle and anterior cerebral arteries caused by the acquisition of control and labeled in different phases of the cardiac cycle
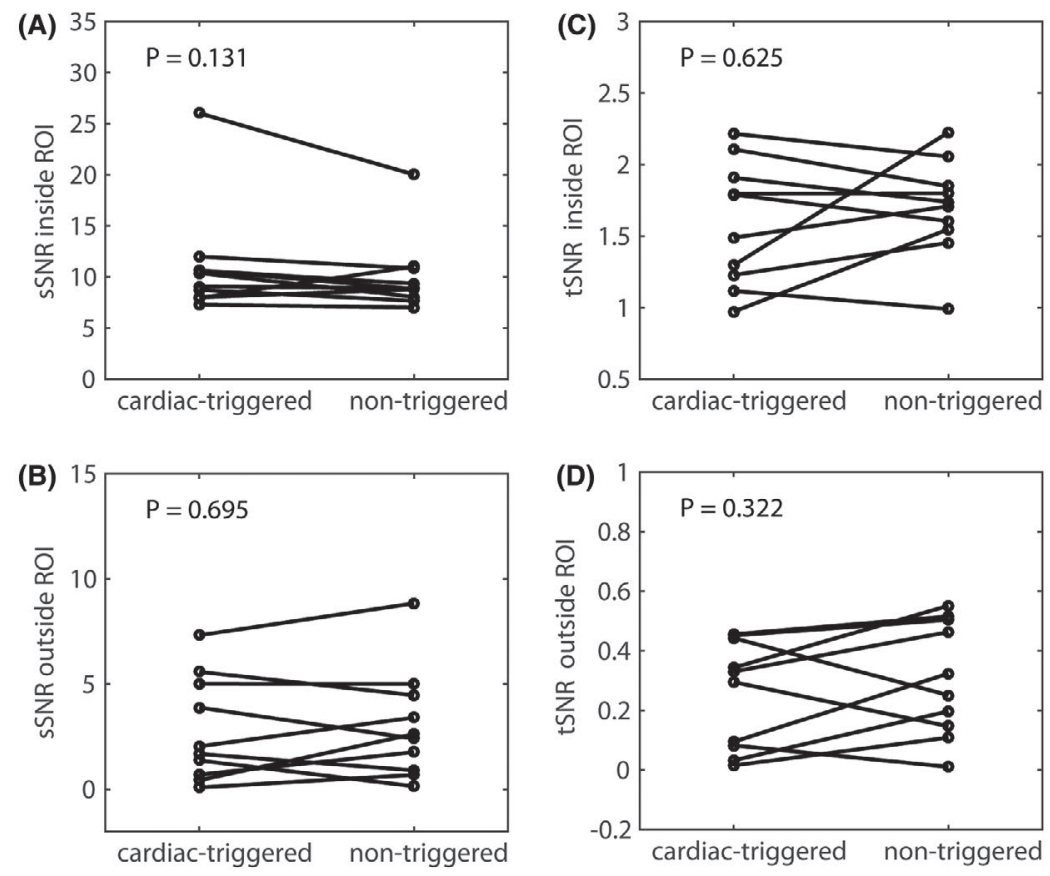

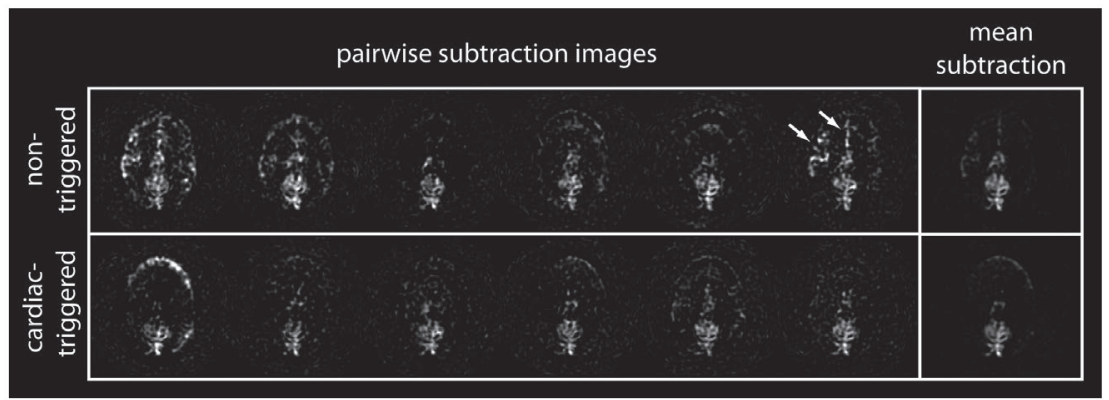

(F)

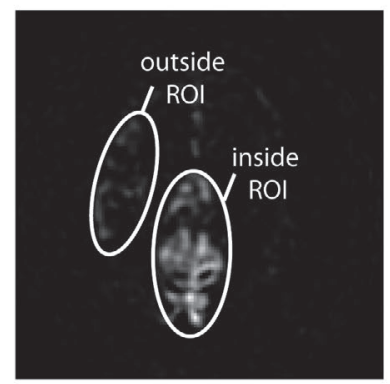

F I G U R E 7 Statistical comparison of cardiac-triggered and nontriggered super-selective pCASL of the dominant VA. A,B, Spatial SNR (sSNR) inside and outside the posterior perfusion territory showed no statistically significant improvement with cardiac triggering. C,D, Cardiac triggering did not result in a significant improvement in the temporal variance, represented by temporal SNR (tSNR). However, there was statistically significant improvement $\left(^{*}\right)$ with cardiac triggering in contrast-to-noise ratio (CNR) (E). F, Inside and outside ROI placement based on mean subtraction perfusion image of the VA

inside or outside the perfusion territory with cardiac triggering. However, there was a statistically significant improvement in contrast-to-noise ratio with cardiac triggering.

\section{3 | Vessel movement}

Figure $8 \mathrm{~A}$ shows the simulated mean labeling efficiency over a range of off-center vessel positions. Both carotid and vertebral arteries experience a steep drop in labeling efficiency for a vessel movement of more than $2 \mathrm{~mm}$ relative to the center of the label spot. The maximum vessel movement measured during the in vivo scanning protocol in study 1 is shown in Figure $8 \mathrm{~B}$. The median maximum movement measured over the group of subjects was below $2 \mathrm{~mm}$, but half of the subjects had at least 1 vessel with more than 2-mm movement during the protocol.

\subsection{Territorial perfusion fraction map}

Building on the results from the first in vivo study, we collected a full set of SS-pCASL images and measured the labeling efficiency in 2 additional subjects in study 2 . The $\mathrm{T}_{1}$ decay-compensated labeling efficiencies and the relative labeling efficiencies of the main feeding arteries in the neck are summarized in Table 1. The relative labeling efficiencies based on the measured signal above the labeling plane and the perfusion signal in the brain tissue showed good agreement for subject 1 . For subject 2, there was an offset between the relative efficiencies. The postprocessed territorial perfusion fraction maps alongside standard color-coded territorial maps are shown in Figure 9. Subject 1 presented with ipsilateral perfusion patterns. The perfusion of the posterior circulation was dominated by the left vertebral artery, 

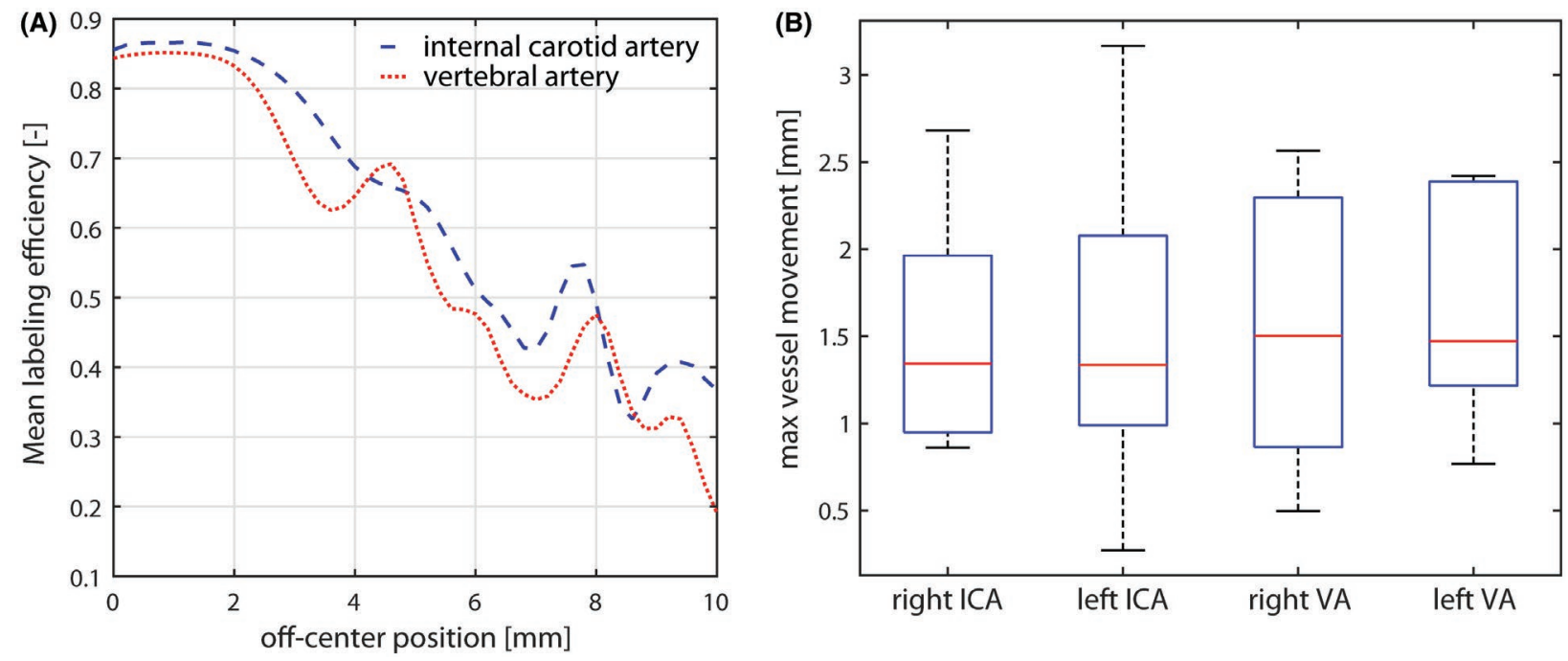

F I G U R E 8 A, Bloch simulation results showing the effect of vessel movement on labeling efficiency for both carotid and vertebral arteries. $\mathrm{B}$, Maximal recorded vessel movement during in vivo scanning protocol over all subjects

TA B L E 1 Labeling efficiencies of the main feeding arteries in the neck

\begin{tabular}{|c|c|c|c|c|c|c|c|c|}
\hline \multirow[b]{2}{*}{ Vessel } & \multicolumn{4}{|c|}{ Subject 1} & \multicolumn{4}{|c|}{ Subject 2} \\
\hline & $\alpha_{\text {true,ns }}$ & $\alpha_{\text {true,vs }}$ & $\alpha_{r e l, a r t}$ & $\alpha_{r e l, t i s s}$ & $\alpha_{\text {true, } n s}$ & $\alpha_{t r u e, v s}$ & $\alpha_{r e l, a r t}$ & $\alpha_{r e l, t i s s}$ \\
\hline Right ICA & 0.79 & 0.48 & 0.60 & 0.63 & 0.80 & 0.35 & 0.44 & 0.28 \\
\hline Left ICA & 0.79 & 0.46 & 0.59 & 0.64 & 0.81 & 0.46 & 0.56 & 0.39 \\
\hline Right VA & 0.53 & 0.40 & 0.75 & - & 0.48 & 0.45 & 0.92 & - \\
\hline Left VA & 0.89 & 0.82 & 0.92 & - & 0.46 & 0.40 & 0.86 & - \\
\hline
\end{tabular}

Note: Measured and $\mathrm{T}_{1}$ decay-compensated labeling efficiencies were based on the nonselective $\left(\alpha_{\text {true,ns }}\right)$ and vessel-selective $\left(\alpha_{\text {true,vs }}\right)$ pCASL scan above the label plane. The relative labeling efficiencies were defined as the ratio of vessel-selective to nonselective labeling efficiencies measured in the arteries $\left(\alpha_{r e l, a r t}\right)$ as well as the ratio of vessel-selective to nonselective perfusion signal at the tissue level $\left(\alpha_{\text {rel }, \text { tiss }}\right)$.

which was of increased caliber as confirmed by the TOF images. Subject 2 presented with an absent basilar artery. Perfusion to the posterior circulation was provided solely by the right ICA artery through the right posterior communicating artery. Again, these findings are in line with the anatomical data.

\section{DISCUSSION AND CONCLUSIONS}

In this paper, we discussed several factors that significantly affect the quality of SS-pCASL acquisitions. We showed that off-resonance compensation, cardiac triggering, a constant clockwise rotation scheme, and vessel location tracking improved labeling efficiency and overall image quality. Furthermore, we presented a workflow to generate accurate territorial perfusion fraction maps by maximizing labeling efficiency and accounting for differences in labeling efficiency between vessels.

\section{1 | Off-resonance calibration}

Study 1 revealed a significant increase in labeling efficiency for both the multiphase and field map compensation strategies. Theoretically, both methods should yield the same optimal phase correction for off-resonance. However, the multiphase calibration consistently yielded higher labeling efficiency and increased perfusion territorial accuracy compared with the field map calibration scans. Although the field map captures the presence of off-resonance due to $\mathrm{B}_{0}$-field inhomogeneity, the multiphase prescan also accounts for additional imperfections in the magnetic field induced by the pCASL label train itself. This was reflected in the linear regression of the off-resonance phase measured with the multiphase prescan and the field map, which showed a constant phase offset between the 2 methods. The most likely source for additional off-resonance is the presence of eddy currents, caused by the fast switching of the slice-selective and vessel-selective gradients. ${ }^{19}$

The multiphase prescan used in this work was derived based on previous work. Jung et al ${ }^{17}$ proposed the acquisition 


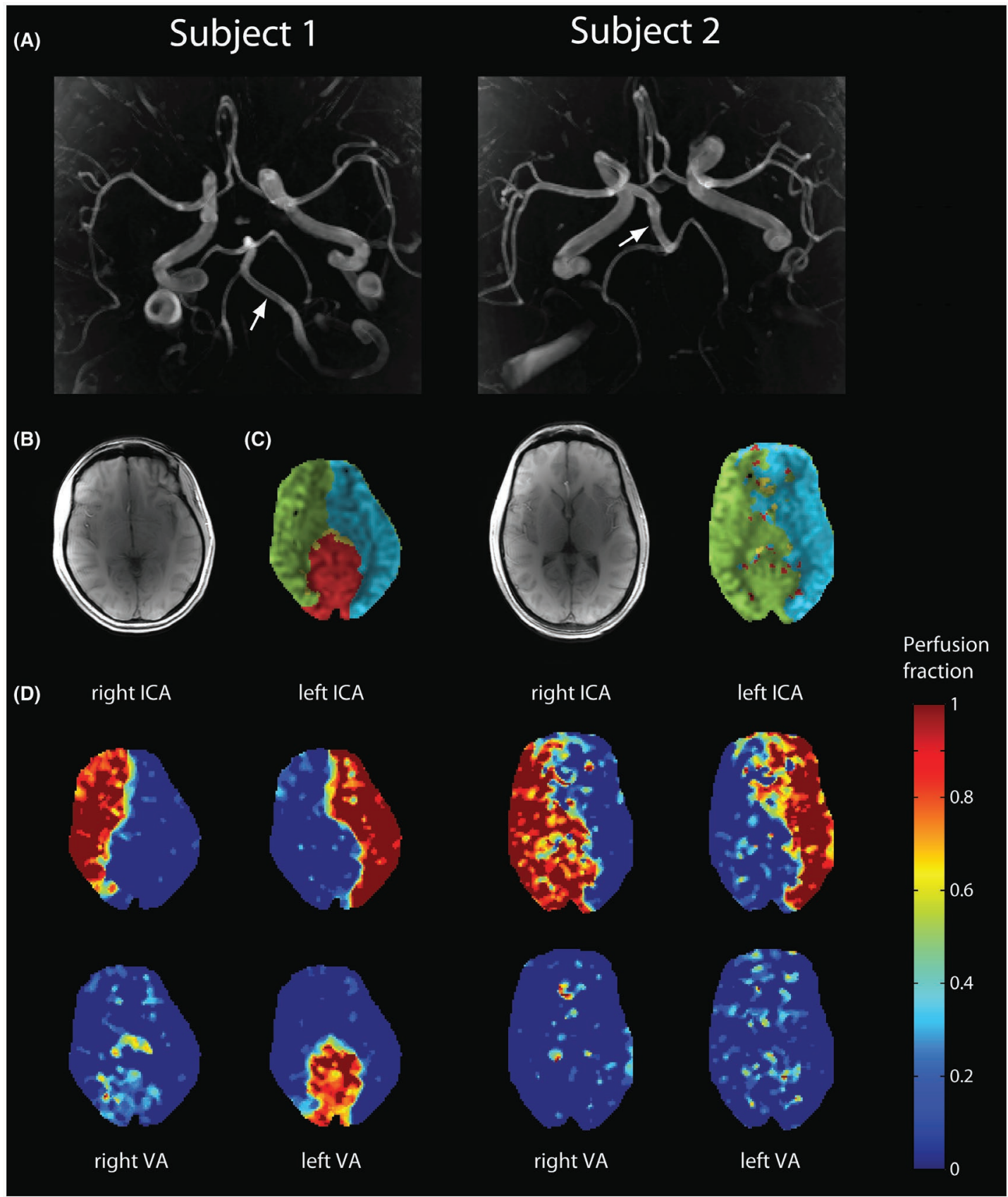

F I G U RE 9 Territorial perfusion in 2 subjects. A, Axial maximum intensity projection of time-of-flight angiogram. B, $\mathrm{T}_{1}$-weighted anatomical image. C, Color-coded perfusion map based on the following color coding: green = right ICA, blue = left ICA, yellow $=$ right VA, red $=$ left VA. D, Territorial perfusion fraction map of the neck arteries. Subject 1 presented with a posterior circulation that was dominantly perfused by the left VA. Time of flight confirms increased caliber of left VA (arrow). Subject 2 presented with absent basilar artery. Posterior circulation is perfused primarily by right ICA (arrow indicates the presence of the right posterior communicating artery with an increased caliber)

of pCASL images over a range of phase increments with subsequent curve fitting to generate the final perfusion images. However, SNR efficiency was reduced due to a small number of averages per phase increment, and the acquisition time was significantly increased. Shin et al introduced a prescan procedure based on a multiphase pCASL acquisition to estimate the ideal phase correction for each vessel. ${ }^{20}$ In addition to the estimation of a global phase correction, the amplitude and direction of in-plane gradients to compensate for off-resonance variations between vessels were calculated. However, this approach required prior knowledge of the vascular territory map. Here, we used a multiphase prescan based on the estimation of an average phase correction over all vessels. In the presence of an off-resonance gradient between vessels, the accuracy of the calibration will be reduced. However, in our in vivo study, differences in off-resonance between vessels were negligible. As an alternative to the multiphase prescan, a field map was used in this work to measure the off-resonance 
in the label plane directly. Jahanian et al previously showed the benefits of estimating the off-resonance compensation in standard pCASL based on a field map. ${ }^{15}$

Despite a notable increase in labeling efficiency, the multiphase calibration has so far not found widespread use in standard pCASL due to the additional time necessary for the acquisition (2:30 minutes) and postprocessing (1 minute) of the calibration scan. However, acquiring only a range of $\pi$ should be sufficient to estimate the optimal phase correction in the future, which would cut the acquisition time in half.

Although nonselective pCASL label schemes typically produce clinically usable images, even in the presence of moderate off-resonance, SS-pCASL label schemes are more susceptible to off-resonance (Figure 2). In vivo experiments showed that in the noncalibrated SS-pCASL scan, labeling efficiency was significantly decreased in some cases to the point where the perfusion signal disappeared in the background noise.

The amount of off-resonance in the labeling plane is highly dependent on the applied shimming procedure. The standard prescan procedure, used in our protocol, only shimmed the image volume without the region of the label plane. While label plane shimming is not available on our system, it might be on other scanners, which could significantly decrease the amount of off-resonance in the label plane and potentially eliminate the need for a calibration scan all together. Furthermore, when the labeling plane is within the shimmed imaged volume (e.g., labeling of intracranial arteries), off-resonance will also be significantly reduced. However, changes in the magnetic field induced by the SS-pCASL label train itself still need to be compensated for. Therefore, the use of a calibration scan for SS-pCASL is highly recommended in situations in which the label plane is outside the shimmed region.

\section{2 | Cardiac triggering}

A qualitative inspection of the mean subtraction images showed a reduction in arterial artifacts and an overall improvement in image quality with cardiac triggering. The statistical comparison showed a significant improvement in contrast-to-noise ratio. However, no significant changes in temporal or spatial SNR were observed. One limiting factor of the analysis was that the temporal variance was based on only 6 pairwise subtractions. The success of cardiac triggering, more specifically the suppression of pulsatility artifacts, is highly dependent on the stability of the heart rate. A change in heart rate between label and control images can lead to imperfect subtractions and therefore artifacts like those present in nontriggered sequences. Movement of the pulse oximeter clip during the scan can further reduce the efficacy. Overall, cardiac triggering increased the scan duration an average of $10 \%$, depending on the heart rate. The effects of the cardiac pulsatility on labeling efficiency were previously studied by Verbree et al. ${ }^{21}$ The triggering of start-oflabeling as well as the end-of-labeling showed no significant change in labeling efficiency. Although cardiac triggering did not improve labeling efficiency, Li et al showed that triggering the start-of-label train reduced temporal variance in nonselective pCASL. ${ }^{18}$ Although pulsatility artifacts are less visible in nonselective pCASL images, they do affect the accuracy of perfusion quantification. In SS-pCASL, pulsatility artifacts have an even larger impact on the image quality and more importantly the estimation of perfusion territories and fractional ratios.

\section{3 | Label rotation scheme}

The constant clockwise rotation of the vessel-selective gradient blips between RF pulses resulted in the highest labeling efficiency for small amounts of off-resonance. For larger offresonance, the pseudo-random rotation scheme, as recommended by Helle et al, ${ }^{7}$ outperformed the constant clockwise rotation scheme. We decided to use the constant clockwise rotation scheme for all in vivo experiments, as off-resonance was compensated. However, in scans without off-resonance calibration, the pseudo-random rotation scheme would be preferable.

\section{4 | Vessel movement}

The success of SS-pCASL depends highly on the match between label spot and vessel position. A label spot size of $18 \mathrm{~mm}$ (FWHM) was chosen based on a number of previous experiments, which showed good selectivity for most vessel anatomies in the neck. Generally, the label spot size should be as large as possible to maximize labeling efficiency, but as small as necessary to avoid labeling of neighboring vessels. For intracranial labeling, the label spot size will have to be reduced by increasing $G_{x y}$ to account for the proximity of cerebral arteries. Based on the label spot size used in our setup, simulations showed a tolerance for a mismatch of approximately $2 \mathrm{~mm}$. These results are in line with the simulation and in vivo results reported in Helle et al. ${ }^{7}$ Here, we wanted to investigate vessel movement under realistic conditions. The acquisition of single-slice 2D TOFs in the label plane allowed us to track vessel movement throughout the protocol. The median maximum movement measured in the group of subjects was below $2 \mathrm{~mm}$, but half of the subjects had at least 1 vessel with more than $2 \mathrm{~mm}$ movement. Movement generally increased toward the end of the scan, as subjects became less comfortable on the table. Vessel movement was only measured within the label plane. Motion in the sliceselection direction was not measured directly in this work. However, because the label plane was positioned in the part of the vasculature where both carotid and vertebral arteries 
are relatively straight, movement in the z-direction was assumed to have only minimal impact on the vessel location.

All subjects in our study were healthy volunteers, capable of lying still for an extended time. In the clinical setting however, these ideal conditions will likely not be met, and the expected vessel movement will most likely be larger. It is therefore recommended to frequently update the vessel position for the SS-pCASL scan throughout the protocol.

\section{5 | Territorial perfusion fraction map}

The overall goal of vessel-selective pCASL is to detect the outline of the perfusion territory and to calculate contributions of individual arteries to the overall perfusion in each voxel. Of particular clinical interest are areas of mixed perfusion. Kansagra et al previously presented a postprocessing method to calculate perfusion fractions of the carotid and basilar perfusion territories based on vessel-encoded pCASL. ${ }^{22}$ Images were scaled based on a relative labeling efficiency (ratio of vessel-selective to nonselective perfusion signal). ${ }^{6,22}$ The method of calculating relative labeling efficiencies was also used in study 1 to compare different offresonance compensation strategies in the left ICA due to its straightforward calculation, without the need for additional scans. Unfortunately, this method is only suitable to estimate the labeling efficiency of the carotid and basilar arteries. Due to the mixing of vertebral flow in the posterior cerebral arteries, the relative efficiency of each vertebral artery cannot be calculated based on the perfusion images alone. Here, we proposed a strategy to measure the labeling efficiency of all 4 vessels directly by acquiring images slightly above the label plane while the inverted spins are still in the arteries. Chen et $\mathrm{al}^{23}$ previously proposed a method to measure the labeling efficiency by tracking the outflow of the bolus in the image plane over time using a Look-Locker acquisition, which was then used to estimate the transit time in each voxel and to compensate for $\mathrm{T}_{1}$ decay. In this work, we acquired a single time point, assuming that the bolus was still fully present in the image plane. The bolus transit time was calculated based on direct measurements of velocity. The labeling efficiency was calculated by first dividing the complex subtraction of control and label image by twice the control image and then compensating for $\mathrm{T}_{1}$ decay during transit. To minimize the acquisition time of the labeling efficiency sequence, we made the assumption that measured signal in the arteries in the control image approximates the fully relaxed spin magnetization. This assumption was based on previous simulations showing that the magnetization in the control state was approximately constant over a wide range of velocities. Furthermore, offresonance was compensated during the acquisition. A comparison of the relative inversion efficiencies in the carotid arteries, obtained in the neck and at the tissue level, showed good agreement in subject 1 but resulted in an offset in subject 2. One possible explanation for this discrepancy is the temporal variation between subtraction images, which is only captured by the relative labeling efficiency based on the perfusion images.

Color-coded perfusion maps provide a straightforward illustration of the perfusion territories of individual arteries. However, it is rather difficult to extract quantitative information on mixed perfusion. Here, we calculated perfusion fractions by scaling the vessel-selective subtraction images based on the labeling efficiencies and dividing by the total perfusion. The scaled and smoothed territorial perfusion fraction maps showed the perfusion territory of each vessel clearly. The results in mixed perfusion territories such as the posterior circulation were in line with the anatomical properties revealed by the corresponding 3D TOF images. For accurate calculation of the perfusion fractions, it is important to ensure that all brain feeding arteries are included in the total perfusion. Here, we carefully inspected the TOF images for any anatomical variations or collateral pathways. Furthermore, we visually compared the total perfusion based on the combined vesselselective images with the nonselective perfusion images.

Vessel-endcoded pCASL has been widely used for mapping the perfusion territories of the main arteries in the neck. While vessel-encoded pCASL requires post-processing to extract the contributions of individual arteries, it is generally thought to be more robust to field inhomogeneity and motion artifacts compared to SS-pCASL due to the use of wider label stripes. However, the presence of these artifacts still reduces the labeling efficiency and accuracy of the measured territorial contributions and should therefore be compensated for.

\subsection{Intracranial labeling}

While our investigation focuses on labeling the main brainfeeding arteries in the neck, SS-pCASL can also be used to label intracranially. Perfusion territory mapping of cerebral arteries can offer valuable clinical information, for example, in patients with intracranial stenosis or arteriovenous malformation. ${ }^{11}$ Many of the discussed issues and solutions may still be applicable; however, there are some important differences to point out. First, with the label plane being within the shimmed region of the brain, the amount of off-resonance will likely be reduced. Second, for intracranial arteries, the tolerance for movement may be different due to the smaller label spot size that is typically chosen to distinguish between neighboring arteries. Finally, pulsatility artifacts due to differences in blood volume during the acquisition will still be present. Therefore, some of the strategies proposed for labeling in the neck could also be beneficial for intracranial applications.

\section{ACKNOWLEDGMENTS}

This work was supported by the German Academic Exchange Service (DAAD), National Institutes of Health (R21EB021562), and the Edward B. Diethrich Professorship. 


\section{ORCID}

Jonas Schollenberger (iD https://orcid.org/0000-0002-9245-3984

C. Alberto Figueroa (iD https://orcid.org/0000-0002-3934-6506

Luis Hernandez-Garcia iD https://orcid.org/0000-0003-3002-0304

\section{REFERENCES}

1. Richter V, Helle M, van Osch M, et al. MR imaging of individual perfusion reorganization using superselective pseudocontinuous arterial spin-labeling in patients with complex extracranial stenoocclusive disease. AJNR Am J Neuroradiol. 2017;38:703-711.

2. Van Laar PJ, Hendrikse J, Mali WPT, et al. Altered flow territories after carotid stenting and carotid endarterectomy. J Vasc Surg. 2007;45:1155-1161.

3. Yaghi S, Bernstein RA, Passman R, Okin PM, Furie KL. Cryptogenic stroke: research and practice. Circ Res. 2017;120:527-540.

4. Kaufmann TJ, Huston J, Mandrekar JN, et al. Complications of diagnostic cerebral angiography. Radiology. 2007;243:812-819.

5. Alsop DC, Detre JA, Golay X, et al. Recommended implementation of arterial spin-labeled perfusion MRI for clinical applications: a consensus of the ISMRM perfusion study group and the european consortium for ASL in dementia. Magn Reson Med. 2014;116:102-116.

6. Wong EC. Vessel-encoded arterial spin-labeling using pseudocontinuous tagging. Magn Reson Med. 2007;58:1086-1091.

7. Helle M, Norris DG, Rüfer S, Alfke K, Jansen O, Van Osch M. Superselective pseudocontinuous arterial spin labeling. Magn Reson Med. 2010;64:777-786.

8. Dai W, Robson PM, Shankaranarayanan A, Alsop DC. Modified pulsed continuous arterial spin labeling for labeling of a single artery. Magn Reson Med. 2010;64:975-982.

9. Liebeskind DS. Collateral circulation. Stroke. 2003;34:2279-2284.

10. Zeng J, Fan Y. Correlation between the integrity of the circle of Willis and the severity of initial noncardiac cerebral infarction and clinical prognosis. Medicine (Baltimore). 2016;95:1-7.

11. Helle M, Rüfer S, van Osch MJP, et al. Superselective arterial spin labeling applied for flow territory mapping in various cerebrovascular diseases. J Magn Reson Imaging. 2013;38:496-503.

12. Lin T, Lai Z, Lv Y, et al. Effective collateral circulation may indicate improved perfusion territory restoration after carotid endarterectomy. Eur Radiol. 2017;1-9.

13. Kaczmarz S, Griese V, Preibisch C, et al. Increased variability of watershed areas in patients with high-grade carotid stenosis. Neuroradiology. 2018;60:311-323.
14. Okell TW, Schmitt P, Bi X, et al. Optimization of 4D vesselselective arterial spin labeling angiography using balanced steady-state free precession and vessel-encoding. NMR Biomed. 2016;29:776-786.

15. Jahanian H, Noll DC, Hernandez-Garcia L. B0 field inhomogeneity considerations in pseudo-continuous arterial spin labeling (pCASL): effects on tagging efficiency and correction strategy. NMR Biomed. 2011;24:1202-1209.

16. Luh WM, Talagala SL, Li TQ, Bandettini PA. Pseudo-continuous arterial spin labeling at $7 \mathrm{~T}$ for human brain: estimation and correction for off-resonance effects using a prescan. Magn Reson Med. 2013;69:402-410.

17. Jung Y, Wong EC, Liu TT. Multiphase pseudocontinuous arterial spin labeling (MP-PCASL) for robust quantification of cerebral blood flow. Magn Reson Med. 2010;64:799-810.

18. Li Y, Mao D, Li Z, et al. Cardiac-triggered pseudo-continuous arterial-spin-labeling: a cost-effective scheme to further enhance the reliability of arterial-spin-labeling MRI. Magn Reson Med. 2018;80:969-975.

19. Guo J, Meakin JA, Jezzard P, Wong EC. An optimized design to reduce eddy current sensitivity in velocity-selective arterial spin labeling using symmetric BIR-8 pulses. Magn Reson Med. 2015;73:1085-1094.

20. Shin DD, Liu TT, Wong EC, Shankaranarayanan A, Jung Y. Pseudocontinuous arterial spin labeling with optimized tagging efficiency. Magn Reson Med. 2012;68:1135-1144.

21. Verbree J, van Osch MJP. Influence of the cardiac cycle on pCASL: cardiac triggering of the end-of-labeling. Magn Reson Mater Phys Biol Med. 2017;1-11.

22. Kansagra AP, Wong EC. Quantitative assessment of mixed cerebral vascular territory supply with vessel encoded arterial spin labeling MRI. Stroke. 2008;39:2980-2985.

23. Chen Z, Zhang X, Yuan C, Zhao X, van Osch M. Measuring the labeling efficiency of pseudocontinuous arterial spin labeling. Magn Reson Med. 2017;77:1841-1852.

How to cite this article: Schollenberger J, Figueroa CA, Nielsen J-F, Hernandez-Garcia L. Practical considerations for territorial perfusion mapping in the cerebral circulation using super-selective pseudocontinuous arterial spin labeling. Magn Reson Med. 2020;83:492-504. https://doi.org/10.1002/mrm.27936 